PROCEEDINGS OF THE

AMERICAN MATHEMATICAL SOCIETY

Volume 127, Number 12, Pages 3643-3652

S 0002-9939(99)04973-4

Article electronically published on May 13, 1999

\title{
NEW PARAMETERS FOR FUCHSIAN GROUPS OF GENUS 2
}

\author{
BERNARD MASKIT
}

(Communicated by Albert Baernstein II)

\begin{abstract}
We give a new real-analytic embedding of the Teichmüller space of closed Riemann surfaces of genus 2 into $\mathbb{R}^{6}$. The parameters are explicitly defined in terms of the underlying hyperbolic geometry. The embedding is accomplished by writing down four matrices in $\operatorname{PSL}(2, \mathbb{R})$, where the entries in these matrices are explicit algebraic functions of the parameters. Explicit inequalities are given to define the image of the embedding; the four matrices corresponding to a point in this image generate a fuchsian group representing a closed Riemann surface of genus 2 .
\end{abstract}

In this note we introduce a new, canonical, real-analytic embedding of the Teichmüller space $\mathcal{T}_{2}$, of closed Riemann surfaces of genus 2 , onto an explicitly defined region $R \subset \mathbb{R}^{6}$. The embedding is defined in terms of the underlying hyperbolic geometry; in particular, the parameters are elementary functions of lengths of simple closed geodesics, and angles and distances between simple closed geodesics.

We start with a specific marked Riemann surface $S_{0}$, and a specific set of normalized (non-standard) generators, $a_{0}, b_{0}, c_{0}, d_{0} \in P S L(2, \mathbb{R})$, for the fuchsian group $G_{0}$ representing $S_{0}$. Then we can realize a point in $\mathcal{T}_{2}$ as a set of appropriately normalized generators $a, b, c, d \in P S L(2, \mathbb{R})$ for the fuchsian group $G$ representing a deformation $S$ of $S_{0}$. We write the entries in the generators, $a, \ldots, d$, as elementary functions of eight parameters, all defined in terms of the underlying hyperbolic geometry, and we write down explicit formulae expressing two of these parameters as functions of the other six. Three of our six parameters are necessarily positive; we give two additional inequalities to obtain necessary and sufficient conditions for the group $G \subset P S L(2, \mathbb{R})$, generated by $a, \ldots, d$, to be an appropriately normalized quasiconformal deformation of $G_{0}$.

There is a related embedding in [3], where the parameters are fixed points of hyperbolic elements of $G$. As in [3], we identify the Teichmüller space with $\mathcal{D} \mathcal{F}$, the identity component of the space of discrete faithful representations of $\pi_{1}\left(S_{0}\right)$ into $\operatorname{PSL}(2, \mathbb{R})$ modulo conjugation. The main differences between these two embeddings is that in [3] the four matrices do not have unit determinant and the parameters there are defined in terms of fixed points of elements of the group; here our matrices do have unit determinant, and the parameters are defined in terms of the underlying hyperbolic geometry.

Received by the editors October 20, 1997 and, in revised form, February 20, 1998.

1991 Mathematics Subject Classification. Primary 30F10; Secondary 32G15.

Research supported in part by NSF Grant DMS 9500557.

(C)1999 American Mathematical Society 
Throughout this paper, unless explicitly state otherwise, all surfaces are closed Riemann surfaces of genus 2, all fuchsian groups are purely hyperbolic, and all references to lengths, distances, etc. are to be understood in terms of hyperbolic geometry.

\section{Chains And NeCKLACES}

A (standard) chain on a surface $S$ is a set of four simple closed non-dividing geodesics, labelled $L_{1}, \ldots, L_{4}$, where $L_{2}$ intersects $L_{1}$ exactly once; $L_{3}$ intersects $L_{2}$ exactly once and is disjoint from $L_{1} ; L_{4}$ intersects $L_{3}$ exactly once and is disjoint from both $L_{1}$ and $L_{2}$. We assume throughout that these geodesics are directed so that, in terms of the homology intersection number, $L_{i} \times L_{i+1}=+1$.

Given the chain $L_{1}, \ldots, L_{4}$ it is easy to see that there are unique simple closed geodesics $L_{5}$ and $L_{6}$ so that $L_{5}$ intersects $L_{4}$ exactly once and is disjoint from $L_{1}$, $L_{2}$ and $L_{3}$; and $L_{6}$ intersects both $L_{5}$ and $L_{1}$ exactly once and is disjoint from the other $L_{i}$. As above, we can assume that these geodesics are also directed so that, using cyclic ordering, $L_{i} \times L_{i+1}=+1$. This set of six geodesics is called a (geodesic) necklace.

The individual geodesics in a chain or necklace are called its links; the points of intersection of the links are called the ties of the necklace. Since the hyperelliptic involution preserves every simple closed geodesic [2], the six ties of a necklace necessarily lie at the six Weierstrass points on the surface (these are the fixed points of the hyperelliptic involution). It follows that the two ties on each link of a necklace divide the link into two arcs of equal length.

If one cuts the surface $S_{0}$ along the geodesics of a chain, one is left with a simply connected subsurface. It follows that we can find elements $a_{0}, b_{0}, c_{0}, d_{0}$ of $\pi_{1}\left(S_{0}\right)$, so that these elements generate $\pi_{1}\left(S_{0}\right)$, and so that the shortest geodesic in the free homotopy class of loops corresponding to, respectively, $a_{0}, b_{0}, c_{0}, d_{0}$, is, respectively, $L_{1}, L_{2}, L_{3}, L_{4}$. There are several possible choices for these elements; we make one such choice in Sect. 3 (see [4] for a theoretical statement of how to make this choice); this yields the one defining relation:

$$
a_{0} b_{0} d_{0} a_{0}^{-1} c_{0}^{-1} d_{0}^{-1} c_{0} b_{0}^{-1}=1 .
$$

We remark that there is a one-to-one correspondence, given by a Nielsen transformation, between these generators and some set of standard generators, $a_{1}, b_{1}, a_{2}, b_{2}$, satisfying the usual relationship:

$$
a_{1} b_{1} a_{1}^{-1} b_{1}^{-1} a_{2} b_{2} a_{2}^{-1} b_{2}^{-1}=1,
$$

given by $a_{0}=a_{1}^{-1}, b_{0}=b_{1}^{-1}, c_{0}=b_{2}^{-1} a_{1}$, and $d_{0}=a_{2}$.

\section{The Teichmüller SPACE}

It is well known that one can identify $\mathcal{T}_{2}$ with the (quasiconformal) deformation space of the fuchsian group $G_{0}$, within the space of fuchsian groups. We will construct our particular set of generators, $a_{0}, b_{0}, c_{0}, d_{0}$ in Sect. ' 3 . These generators will be normalized so that $c_{0}$ has its repelling fixed point at 0 , and its attracting fixed point at $\infty$; the attracting fixed point of $a_{0}$ is positive and less than 1 ; and the product of the fixed points of $a_{0}$ is equal to 1 .

A point in $\mathcal{T}_{2}$ can be regarded as being an equivalence class of orientationpreserving homeomorphisms $f$ of the closed upper half-plane onto itself, together 
with representations $\phi: G_{0} \rightarrow P S L(2, \mathbb{R})$, where, for all $g \in G_{0}$, and for all $z$ in the upper half-plane, $f \circ g(z)=\phi(g) \circ f(z)$. Two such homeomorphisms are equivalent if the corresponding representations are equivalent; two such representations, $\phi$ and $\phi^{\prime}$ are equivalent if there is an element $m \in P S L(2, \mathbb{R})$ so that $m \phi m^{-1}=\phi^{\prime}$.

Once we have defined $G_{0}$, then we define the normalized deformation space $\mathcal{D F}$ as the space of representations $\phi: G_{0} \rightarrow \operatorname{PSL}(2, \mathbb{R})$, where the representation $\phi$ is faithful; the image group $G=\phi\left(G_{0}\right)$ is discrete, with $S=\mathbb{H}^{2} / G$ a closed Riemann surface of genus 2; the product of the fixed points of $a=\phi\left(a_{0}\right)$ is equal to 1 , with the attracting fixed point positive and smaller than the repelling fixed point; the repelling fixed point of $c=\phi\left(c_{0}\right)$ is at 0 ; and the attracting fixed point of $c$ is at $\infty$.

The normalizations given in the definition of $\mathcal{D} \mathcal{F}$ make it clear that there is a well defined bijection $\Phi: \mathcal{T}_{2} \rightarrow \mathcal{D F}$. It is well known that, in appropriate coordinates, $\Phi$ is a real-analytic diffeomorphism.

\section{The Base SuRface}

Our base point $G_{0}$ is a subgroup of the $(2,4,6)$-triangle group. As a hyperelliptic surface, the underlying Riemann surface is the double cover of the sphere with branch points at the sixth roots of unity. We label these, in natural order, as $\omega_{1}=e^{\pi i / 3}, \ldots, \omega_{5}=e^{5 \pi i / 3}, \omega_{6}=1$. Regarding these as cyclically ordered, one can construct a geodesic necklace as follows: $L_{i}$ is the arc of the unit circle from $\omega_{i}$ to $\omega_{i+1}$ on the upper sheet, followed by the arc of the unit circle from $\omega_{i+1}$ back to $\omega_{i}$ on the lower sheet. Using reflection in the unit circle, it is easy to see that $L_{1}, \ldots, L_{6}$ are all geodesics. Also, they all have the same length; and they all meet at right angles. This necklace divides $S_{0}$ into four equilateral hexagons, with all right angles.

We label these hexagons as $H_{1}, \ldots, H_{4}$. We note that the hyperelliptic involution acts on these; we can assume that we have labelled the hexagons so that the hyperelliptic involution interchanges $H_{1}$ and $H_{3}$, and also interchanges $H_{2}$ and $H_{4}$.

We label the sides of $H_{1}\left(H_{2}\right)$ as $A_{0}, B_{0}, C_{0}, D_{0}, E_{0}, F_{0}\left(A_{0}^{\prime}, B_{0}^{\prime}, C_{0}^{\prime}, D_{0}^{\prime}, E_{0}^{\prime}, F_{0}^{\prime}\right)$, where $A_{0}\left(A_{0}^{\prime}\right)$ is an arc of $L_{1}, B_{0}\left(B_{0}^{\prime}\right)$ is an arc of $L_{2}$, etc. We choose the hexagons $H_{1}$ and $H_{2}$ so that $A_{0}$ and $A_{0}^{\prime}$ are the same arc of $L_{1}$; then, likewise $C_{0}$ and $C_{0}^{\prime}$ are the same arc of $L_{3}$, and $E_{0}$ and $E_{0}^{\prime}$ are the same arc of $L_{5}$. Since both arcs of each $L_{i}$ have equal length, it follows that $B_{0}$ and $B_{0}^{\prime}$ have equal length, as do $D_{0}$ and $D_{0}^{\prime}$, and $F_{0}$ and $F_{0}^{\prime}$. We orient $A_{0}$ so that it points towards $B_{0}$; this induces an orientation on all the sides of $H_{1}$.

We normalize $G_{0}$, the fuchsian group acting on $\mathbb{H}^{2}$ and representing $S_{0}$, so that a lift of $C_{0}$ lies on the imaginary axis, with the vertical as its positive direction, and so that the point of intersection of $C_{0}$ with the common orthogonal between $A_{0}$ and $C_{0}$ lies at the point $i$. (The choice of language is deliberate; in this particular group, $B_{0}$ is the common orthogonal between $A_{0}$ and $C_{0}$, but that identification will not persist under deformation.) We lift $H_{1}$ and $H_{2}$ so that $C_{0}$ and $C_{0}^{\prime}$ have the same lift. Then $B_{0}$ and $B_{0}^{\prime}$ lie on the same line in the upper half-plane; also $D_{0}$ and $D_{0}^{\prime}$ lie on the same line. We now make an assumption regarding orientation: If necessary, we interchange the labels on $H_{1}$ and $H_{2}$ so that this lift of $H_{1}$ lies in the right half-plane.

We note that the sides $A_{0}, \ldots, F_{0}$ are oriented. We define the elements $a_{0}, b_{0}, c_{0}$, $d_{0}$, respectively, as the hyperbolic transformation whose axis contains, respectively, 
$A_{0}, B_{0}, C_{0}, D_{0}$, and whose translation length in the positive direction along this axis is, respectively, $2\left|A_{0}\right|, 2\left|B_{0}\right|, 2\left|C_{0}\right|, 2\left|D_{0}\right|$ (these are of course all equal). These elements are all in $G_{0}$; we will see below that they generate $G_{0}$.

One could use the fact that $G_{0}$ is a subgroup of the $(2,4,6)$-triangle group to calculate the corresponding multipliers or traces for $a_{0}, \ldots, d_{0}$; this calculation is most easily made by using the unit disc model of the hyperbolic plane. Instead, we will write down explicit matrices $\tilde{a}_{0}, \ldots, \tilde{d}_{0} \in S L(2, \mathbb{R})$, and show that the corresponding elements $a_{0}, \ldots, d_{0} \in P S L(2, \mathbb{R})$ have the requisite properties. We set

$$
\begin{gathered}
\tilde{a}_{0}=\left(\begin{array}{cc}
2-2 \sqrt{3} & 3 \\
-3 & 2+2 \sqrt{3}
\end{array}\right), \quad \tilde{b}_{0}=\left(\begin{array}{cc}
2 & \sqrt{3} \\
\sqrt{3} & 2
\end{array}\right) \\
\tilde{c}_{0}=\left(\begin{array}{cc}
2+\sqrt{3} & 0 \\
0 & 2-\sqrt{3}
\end{array}\right), \quad \tilde{d}_{0}=\left(\begin{array}{cc}
2 & -3-2 \sqrt{3} \\
3-2 \sqrt{3} & 2
\end{array}\right) .
\end{gathered}
$$

We need to prove that the group $G_{0}$, generated by $a_{0}, \ldots, d_{0}$, is appropriately normalized, discrete, purely hyperbolic and represents our surface $S_{0}$, as described above. We remark that it would suffice for our purposes to show that $G_{0}$ is appropriately normalized, discrete, purely hyperbolic, and represents some surface of genus 2, which we could then take to be our base surface.

We first observe that $c_{0}$ has its attracting fixed point at $\infty$, and its repelling fixed point at 0 . We also easily observe that the fixed points of $a_{0}$ are both positive, with product equal to 1 , and that the attracting fixed point is smaller than the repelling fixed point.

We also observe that $\tilde{a}_{0}, \ldots, \tilde{d}_{0}$ all have the same trace equal to 4 .

Before proceeding, we need the following well-known observation (a proof can be found in [1]). Let $a$ and $c$ be hyperbolic Möbius transformations acting on the upper half-plane. Assume that the axes of $a$ and $c$ do not intersect, even at the circle at infinity. Let $J=\langle a, c\rangle .{ }^{1}$ We say that $a$ and $c$ are geometric generators of $J$ if the axis of $a$, respectively, $c$, bounds a half-plane that is precisely invariant under $\langle a\rangle$, respectively, $\langle c\rangle$. Assume that the axes of $a$ and $c$ are oriented (the direction is of course given by the transformations) so that the region between these axes lies on the left for both axes, or lies on the right for both axes. Assume also that we have chosen corresponding matrices $\tilde{a}$ and $\tilde{c}$ in $S L(2, \mathbb{R})$, with positive trace. Then $a$ and $c$ are geometric generators of $J$ if and only if the trace of $\tilde{e}^{-1}=\tilde{c} \tilde{a}$ is less than -2 .

Let $J_{1}=\left\langle a_{0}, c_{0}\right\rangle$. We compute the trace of $\tilde{e}_{0}^{-1}=\tilde{c}_{0} \tilde{a}_{0}$, and observe that it is equal to -4 .

We set $r_{0}(z)=-\bar{z}$, and represent it by the matrix

$$
\tilde{r}_{0}=\tilde{r}_{0}^{-1}=\left(\begin{array}{cc}
-1 & 0 \\
0 & 1
\end{array}\right) .
$$

We set $\tilde{a}_{0}^{\prime}=\tilde{r}_{0} \tilde{a}_{0} \tilde{r}_{0}^{-1}$ and we set $\tilde{e}_{0}^{\prime}=\tilde{r}_{0} \tilde{e}_{0} \tilde{r}_{0}^{-1}$; we note that $\tilde{a}_{0}^{\prime}$ has positive trace and that $\tilde{e}_{0}^{\prime}$ has negative trace. Easy computations now show that $\tilde{a}_{0}^{\prime}=\tilde{b}_{0}^{-1} \tilde{a}_{0} \tilde{b}_{0}$ and $\left(\tilde{e}_{0}^{\prime}\right)^{-1}=\tilde{d}_{0} \tilde{c}_{0} \tilde{a}_{0} \tilde{d}_{0}^{-1}$.

Let $J_{2}=\left\langle a_{0}^{\prime}, c_{0}\right\rangle$. Note that the left half-plane is precisely invariant under $\left\langle c_{0}\right\rangle$ in $J_{1}$, while the right half-plane is precisely invariant under $\left\langle c_{0}\right\rangle$ in $J_{2}$. Hence we can use combination theorems, as in [3], to amalgamate $J_{1}$ to $J_{2}$, to obtain a new

\footnotetext{
${ }^{1}$ We use $\langle A, B, \ldots\rangle$ to denote the group generated by $A, B, \ldots$.
} 
group, call it $J_{3}$, representing a sphere with four holes. Since all four primitive boundary elements, $a_{0}, e_{0}, a_{0}^{\prime}, e_{0}^{\prime}$ have the same trace, all four holes have the same size. Since $b_{0}$ conjugates $a_{0}^{\prime}$ into $a_{0}$, we can use combination theorems to adjoin $b_{0}$ to $J_{3}$, to obtain a new purely hyperbolic discrete group, call it $J_{4}$, representing a torus with two equal sized holes. Finally, since $d_{0}$ conjugates $e_{0}$ into $e_{0}^{\prime}$, we again use combination theorems to adjoin $d_{0}$ to $J_{4}$, and thus obtain the discrete purely hyperbolic group $G_{0}$, representing a closed Riemann surface of genus 2 .

We also note that, as a consequence of the combination theorems, we obtain that these generators have equation (1) as their one defining relation.

3.1. Connection with the $(2,4,6)$-triangle group. For the sake of completeness, we prove here that $G_{0}$ is indeed a subgroup of the $(2,4,6)$-triangle group, and that our generators do indeed have the desired properties; that is, the elements $a_{0}, b_{0}, c_{0}, d_{0}, e_{0}=\left(c_{0} a_{0}\right)^{-1}, f_{0}=\left(b_{0} d_{0}\right)^{-1}$ all have equal traces; and their axes are either disjoint or meet at right angles to form the hexagon $A_{0}, B_{0}, C_{0}, D_{0}, E_{0}, F_{0}$.

It is easy to compute the trace of $\tilde{f}_{0}=\left(\tilde{b}_{0} \tilde{d}_{0}\right)^{-1}$, and observe that it is equal to -4 .

The axis of $c_{0}$ lies on the imaginary axis; it is easy to see that the axes of $b_{0}$ and $d_{0}$ are orthogonal to it, and that the other axes are disjoint from it. In order to prove that each other pair of axes is either disjoint or orthogonal, as the case may be, we make the following observation. If

$$
\tilde{a}=\left(\begin{array}{ll}
\alpha & \beta \\
\gamma & \delta
\end{array}\right)
$$

is a matrix representing a hyperbolic transformation, with fixed points at neither 0 nor $\infty$, acting on the upper half-plane, then

$$
\tilde{r}_{a}=\frac{1}{\sqrt{(\alpha+\delta)^{2}-4}}\left(\begin{array}{cc}
\alpha-\delta & 2 \beta \\
2 \gamma & \delta-\alpha
\end{array}\right)
$$

represents the reflection whose fixed points are along the axis of $a$. The axes of $a$ and $b$ are orthogonal if and only if the trace of $\tilde{r}_{a} \tilde{r}_{b}=0$; the axes of $a$ and $b$ are disjoint, even at the circle at infinity, if and only if the absolute value of the trace of $\tilde{r}_{a} \tilde{r}_{b}$ is greater than 2 .

After doing the above computations, we see that the axes of $a_{0}, \ldots, f_{0}$ divide $S_{0}$ into four right angle equilateral hexagons. Since the equilateral hexagon with all right angles is unique, it follows that our group and generators are as desired. One could also observe that these hexagons, and hence also $S_{0}$, admit an action by a rotation of order 6 ; the quotient of $S_{0}$ by this action is the orbifold of signature $(0,3 ; 2,4,6)$.

\section{Deformations}

Let $\phi: G_{0} \rightarrow P S L(2, \mathbb{R})$ be a deformation in $\mathcal{D F}$. We define $a, \ldots, f$ by $a=$ $\phi\left(a_{0}\right), \ldots, f=\phi\left(f_{0}\right)$, and let $G=\phi\left(G_{0}\right)$. Since $\phi$ can be realized by an orientationpreserving homeomorphism of the closed disc, the axes of $a, \ldots, f$ form a hexagon, $H$, and the axes of $a$ and $c$ do not meet, even on the circle at infinity. Also $G$ is normalized so that the axis of $c$ lies on the imaginary axis, with 0 as the repelling fixed point, so that the point of intersection of the axis of $c$ with the common orthogonal to the axes of $a$ and $c$ lies at the point $i$ (given the orientation, and given that the axis of $c$ lies on the imaginary axis, this is equivalent to saying that 
the product of the fixed points of $a$ is equal to 1 ). We observe that $H$ necessarily lies in the right half-plane. We let $A, \ldots, F$ be the sides of $H$, where $A$ lies on the axis of $a$, etc. We orient each side so that its orientation agrees with that of the positive direction of the corresponding hyperbolic Möbius transformation.

The axes of $a, \ldots, f$ form a geodesic necklace on the underlying Riemann surface $S=\mathbb{H}^{2} / G$. This necklace divides $S$ into four hexagons, which we can label as $H_{1}, \ldots, H_{4}$, where $H_{1}$ is the projection of $H$, and $H_{2}$ is the projection of $H^{\prime}$, the hexagon in the left half-plane, having $C$ as one of its sides. As above, we choose the labels so that the hyperelliptic involution interchanges $H_{1}$ and $H_{3}$, and also interchanges $\mathrm{H}_{2}$ and $\mathrm{H}_{4}$.

We have the sides of $H_{1}$ labelled as $A, \ldots, F$; we can label the corresponding sides of $H_{2}$ as $A^{\prime}, \ldots, F^{\prime}$, so that $A$ and $A^{\prime}$ have the same length, as do $B$ and $B^{\prime}$, etc. We label the angles of $H_{1}\left(H_{2}\right)$ in order as $\theta_{1}, \ldots, \theta_{6}\left(\theta_{1}^{\prime}, \ldots, \theta_{6}^{\prime}\right)$, where $\theta_{1}$ $\left(\theta_{1}^{\prime}\right)$ lies between $A$ and $B\left(A^{\prime}\right.$ and $\left.B^{\prime}\right)$. Since the four hexagons fit together on $S$, it follows that, for each $i=1, \ldots, 6, \theta_{i}+\theta_{i}^{\prime}=\pi$.

\section{Parameters}

In this section, we consider a general deformation $\phi \in \mathcal{D F}$; we set $(a, b, c, d)=$ $\left(\phi\left(a_{0}\right), \phi\left(b_{0}\right), \phi\left(c_{0}\right), \phi\left(d_{0}\right)\right)$. We define our eight basic parameters, and we write down matrices $(\tilde{a}, \tilde{b}, \tilde{c}, \tilde{d})$, representing $(a, b, c, d$, ), where the entries in these matrices are particular functions of these parameters.

5.1. Geometric meaning of the parameters. Define $e=a^{-1} c^{-1}$, and $f=$ $d^{-1} b^{-1}$. Then, since $\phi$ is a deformation, the axes of $a, \ldots, f$ form a hexagon $H$, with sides $A, \ldots, F$, where $A$ lies on the axis of $a$, etc. Let $G=\langle a, b, c, d\rangle$, and let $S=\mathbb{H}^{2} / G$.

Our basic parameters are $\alpha, \beta, \gamma, \delta, \mu, \sigma, \tau$ and $\rho$, defined as follows. Set $\alpha=|A|, \beta=|B|, \gamma=|C|, \delta=|D|$. Let $L$ be the common orthogonal between the axes of $a$ and $c$. Let $\mu^{\prime}=|L|$; define $\mu$ by $\operatorname{coth} \mu=\cosh \mu^{\prime}$. Let $\sigma$ be the distance, measured in the positive direction along the axis of $c$, between $L$ and the point where the axis of $b$ crosses the axis of $c$. Let $\theta_{2}$ be the angle inside $H$ between the axes of $b$ and $c$; and let $\theta_{3}$ be the angle inside $H$ between the axes of $c$ and $d$. Define $\tau$ and $\rho$ by $\tanh \tau=\cos \theta_{2}$ and $\tanh \rho=-\cos \theta_{3}$.

We note that $\alpha, \beta, \gamma, \delta$ and $\mu$ are necessarily positive.

We define $\tilde{a}, \ldots, \tilde{d}$ as being the matrices in $S L(2, \mathbb{R})$, with positive trace, representing, respectively, $a, \ldots, d$.

Proposition 5.1. We have the following representations.

$$
\begin{gathered}
\tilde{a}=\frac{1}{\sinh \mu}\left(\begin{array}{cc}
\sinh (\mu-\alpha) & \sinh \alpha \\
-\sinh \alpha & \sinh (\mu+\alpha)
\end{array}\right), \tilde{b}=\frac{1}{\cosh \tau}\left(\begin{array}{cc}
\cosh (\tau+\beta) & e^{\sigma} \sinh \beta \\
e^{-\sigma} \sinh \beta & \cosh (\tau-\beta)
\end{array}\right), \\
\tilde{c}=\left(\begin{array}{cc}
e^{\gamma} & 0 \\
0 & e^{-\gamma}
\end{array}\right), \tilde{d}=\frac{1}{\cosh \rho}\left(\begin{array}{cc}
\cosh (\rho-\delta) & -e^{\sigma+\gamma} \sinh \delta \\
-e^{-\sigma-\gamma} \sinh \delta & \cosh (\rho+\delta)
\end{array}\right) .
\end{gathered}
$$

Proof. Since the projection of the axes of $a_{0} \ldots, f_{0}$, form a geodesic necklace on $S_{0}$, the projection of the axes of $a, \ldots, f$ form a geodesic necklace on $S$. Since $S$ is hyperelliptic, this necklace is evenly spaced. Hence, for example, the length of $A$ is half the length of the closed geodesic formed by the projection of the axis of $a$; it follows that $\operatorname{trace}(\tilde{a})=2 \cosh \alpha ; \operatorname{trace}(\tilde{b})=2 \cosh \beta ; \operatorname{trace}(\tilde{c})=2 \cosh \gamma$ and $\operatorname{trace}(\tilde{d})=2 \cosh \delta$. 
We easily compute that the matrices above all have unit determinant; hence the expressions above for $\tilde{a}, \ldots, \tilde{d}$ all have correct determinant and correct trace.

Since we have normalized $c$ so that its repelling fixed point is at 0 , and its attracting fixed point is at $\infty$, the above expression for $\tilde{c}$ is correct.

It follows from our normalization that the common orthogonal $L$ between the axes of $a$ and $c$ intersects the axis of $c$ at the point $i$, with the attracting fixed point of $a$ positive and smaller than the repelling fixed point; hence there is a number $\tilde{\mu}>0$ so that the repelling fixed point of $a$ is at $e^{\tilde{\mu}}$ and the attracting fixed point of $a$ is at $e^{-\tilde{\mu}}$. Let $\psi$ denote the argument of the euclidean ray from the origin tangent to the axis of $a$. An easy computation shows that $\cosh \mu^{\prime}=\csc \psi=\operatorname{coth} \tilde{\mu}$, from which it follows that $\tilde{\mu}=\mu$. It is easy to check that $\tilde{a}$, regarded as a Möbius transformation, has its attracting fixed point at $e^{-\mu}$ and its repelling fixed point at $e^{\mu}$. Hence the expression for $\tilde{a}$ is correct.

We defined $\sigma$ to be the distance, measured along the axis of $c$, in the positive direction, between the point of intersection with $L$, which has been normalized to be at the point $i$, and the point of intersection with the axis of $b$. Then the axis of $b$ crosses the imaginary axis at the point $i e^{\sigma}$; hence there is a number $\tilde{\tau}>0$ so that the attracting fixed point of $b$ is at $e^{\sigma+\tilde{\tau}}$ and the repelling fixed point is at $-e^{\sigma-\tilde{\tau}}$. Easy computations show that $\tilde{\tau}$ and $\theta_{2}$ are related by $\tanh \tilde{\tau}=\cos \theta_{2}$. Hence $\tilde{\tau}=\tau$. It is easy to check that $\tilde{b}$, regarded as a Möbius transformation, has the desired fixed points.

We observed above that the distance, along the axis of $c$, between the point of intersection with the axis of $b$ and the point of intersection with the axis of $d$ must be half the translation length of $c$. Hence this point of intersection is the point $i e^{\sigma+\gamma}$. We conclude that there is a number $\tilde{\rho}>0$ so that the repelling fixed point of $d$ is at $e^{\sigma+\gamma+\tilde{\rho}}$, and the attracting fixed point is at $-e^{\sigma+\gamma-\tilde{\rho}}$. Then $\tilde{\rho}$ and $\theta_{3}$ are related by $\tanh \tilde{\rho}=-\cos \theta_{3}$, from which we conclude that $\tilde{\rho}=\rho$. We observe that $\tilde{d}$, regarded as a Möbius transformation, has the desired fixed points.

Proposition 5.2. The parameters, $\sinh \alpha, \sinh \beta, \sinh \gamma, \sinh \delta, \sinh \mu, \sinh \sigma$, $\sinh \tau, \sinh \rho$, depend algebraically on the point $\phi \in \mathcal{D} \mathcal{F}$.

Proof. Observe that $2 \cosh \alpha=\operatorname{trace}(a) ; 2 \cosh \beta=\operatorname{trace}(b) ; 2 \cosh \gamma=\operatorname{trace}(c)$; $2 \cosh \delta=\operatorname{trace}(d) ; 2 \cosh \mu$ is the sum of the fixed points of $a ; e^{2 \sigma}$ is the product of the fixed points of $b ; 2 e^{\sigma} \sinh \tau$ is the sum of the fixed points of $b$; and $2 e^{\sigma+\gamma} \sinh \rho$ is the sum of the fixed points of $d$.

5.2. Remark. We also remark that the entries in the matrices $\tilde{a}, \tilde{b}, \tilde{c}, \tilde{d}$ are algebraic functions of the parameters, $\sinh \alpha, \sinh \beta, \sinh \gamma, \sinh \delta, \sinh \mu, \sinh \sigma, \sinh \tau, \sinh \rho$.

5.3. The parameter map. We will see below that $\sinh \mu$ and $\sinh \delta$ can be written as algebraic functions of the other parameters. We define the map $\Psi: \mathcal{D F} \rightarrow \mathbb{R}^{6}$, by $\Psi(\phi)=(\sinh \alpha, \sinh \beta, \sinh \gamma, \sinh \sigma, \sinh \tau, \sinh \rho)$.

\section{NeCESSARy CONDitions}

In this section, we assume that the matrices, $\tilde{a}, \ldots, \tilde{d}$, are defined by the formulae in 5.1 as functions of the eight parameters, $\alpha, \ldots, \rho$. We denote the corresponding Möbius transformations by $a, \ldots, d$. We assume that there is a deformation $\phi \in$ $\mathcal{D} \mathcal{F}$, so that $(a, \ldots, d)=\left(\phi\left(a_{0}\right), \ldots, \phi\left(d_{0}\right)\right)$. We also explicitly assume that $\mu>0$. Let $G=\phi\left(G_{0}\right)$. 
We have normalized $c$ so that 0 is the repelling fixed point; this means that $\gamma>0$. It also follows from our normalization that the attracting fixed point of $b$ is positive; it follows that $\beta>0$. We have normalized $a$ so that its attracting fixed point lies between 0 and 1 ; this, together with our assumption that $\mu>0$, implies that $\alpha>0$.

We explicitly state these three inequalities as our first condition.

$$
\alpha>0, \quad \beta>0, \quad \gamma>0 .
$$

For future use, we remark that it also follows from our normalization that the attracting fixed point of $d$ is negative; this implies that $\delta>0$.

Since $a_{0}$ and $c_{0}$ are geometric generators for the subgroup $J_{1}$ they generate; it follows that $a$ and $c$ are geometric generators for the subgroup $K_{1}$ that they generate. By the criterion in [1] mentioned above, it follows that the trace of the matrix $\tilde{e}^{-1}=\tilde{c} \tilde{a}$ is less than -2 . This yields our first non-trivial inequality:

$$
\operatorname{coth} \mu>\frac{1+\cosh \alpha \cosh \gamma}{\sinh \alpha \sinh \gamma} .
$$

We next define the matrix $\tilde{a}^{\prime}$ by $\tilde{a}^{\prime}=\tilde{b}^{-1} \tilde{a} \tilde{b}$, and we let $K_{2}$ be the subgroup of $G$ generated by the corresponding $a^{\prime}$ and $c$. We also set $\tilde{e}^{\prime}=\left(\tilde{a}^{\prime}\right)^{-1} \tilde{c}^{-1}$, and note that, since $\phi$ is an isomorphism, the corresponding elements of $P S L(2, \mathbb{R})$ satisfy $e^{\prime}=\operatorname{ded}^{-1}$.

We note that the projection of the axes of $a, c$ and $e$ to $S=\mathbb{H}^{2} / G$ consists of three simple disjoint non-dividing geodesics. These split $S$ into two isometric pairs of pants.

Following the known construction of $G_{0}$, and using the fact that $\phi$ is a deformation, we see that these pairs of pants can be realized as $\mathbb{H}^{2} / K_{1}$ and $\mathbb{H}^{2} / K_{2}$, where the left half-plane (right half-plane) is precisely invariant under $\langle c\rangle$ in $K_{1}$ $\left(K_{2}\right)$. Also, since these two pairs of pants are isometric, there is an orientationreversing isometry of $\mathbb{H}^{2}$, interchanging the left and right half-planes, commuting with $c$ and conjugating $K_{1}$ onto $K_{2}$. Such a transformation necessarily has the form $r(z)=-\nu \bar{z}, \nu>0$.

We choose the following matrices to represent $r$ and $r^{-1}$ :

$$
\tilde{r}=\left(\begin{array}{cc}
-\nu & 0 \\
0 & 1
\end{array}\right), \quad \tilde{r}^{-1}=\left(\begin{array}{cc}
-\nu^{-1} & 0 \\
0 & 1
\end{array}\right) .
$$

There are still many choices for $\nu$. We choose $\nu$ so that $r$ conjugates $a$ to $a^{\prime}$; i.e., $a^{\prime}=r a r^{-1}$. Then, since $a^{\prime}=b^{-1} a b$, we obtain

$$
b r a r^{-1}=a b .
$$

Of course, we know (4) only as an equation in $\operatorname{PSL}(2 ; \mathbb{R})$. However, since the matrices $\tilde{b} \tilde{r} \tilde{a} \tilde{r}^{-1}$ and $\tilde{a} \tilde{b}$ have unit determinant, there are only two possibilities in $S L(2, \mathbb{R})$; i.e.,

$$
\tilde{b} \tilde{r} \tilde{a} \tilde{r}^{-1}= \pm \tilde{a} \tilde{b} .
$$

Since $\nu$ is a ratio of fixed points, it is well defined as a function on $\mathcal{D F}$. It follows that, for any choice of matrices $\tilde{a}$ and $\tilde{b}$, representing $a$ and $b$, respectively, the trace of $\tilde{b} \tilde{r} \tilde{a} \tilde{r}^{-1} \tilde{b}^{-1} \tilde{a}^{-1}$ is a well defined function on $\mathcal{D} \mathcal{F}$, which is connected. We conclude that the same sign in (5) must hold throughout $\mathcal{D F}$. Since $\tilde{b}_{0} \tilde{r}_{0} \tilde{a}_{0} \tilde{r}_{0}^{-1}=\tilde{a}_{0} \tilde{b}_{0}$, we 
obtain that

$$
\tilde{b} \tilde{r} \tilde{a} \tilde{r}^{-1}=\tilde{a} \tilde{b} .
$$

The four equations for the entries in (6) reduce to only two equations in our parameters. The diagonal terms both yield

$$
\nu=e^{2 \sigma} .
$$

Substituting this value for $\nu$ in either equation obtained from the off diagonal terms, we obtain an equation that can be solved for $\cosh \mu$. We combine this with our basic assumption about the positivity of $\mu$ to obtain the following.

$$
\cosh \mu=\operatorname{coth} \beta \cosh \sigma \cosh \tau+\sinh \sigma \sinh \tau, \quad \mu>0 .
$$

Since $r$ commutes with $c$, we have $r e r^{-1}=r a^{-1} c^{-1} r^{-1}=\left(a^{\prime}\right)^{-1} c^{-1}=e^{\prime}=$ $d e d^{-1}=d a^{-1} c^{-1} d^{-1}$. From this, we obtain

$$
d c a=\text { rcar }^{-1} d .
$$

As above, $(9)$ yields two possible equations in $S L(2, \mathbb{R})$ :

$$
\tilde{d} \tilde{c} \tilde{a}= \pm \tilde{r} \tilde{c} \tilde{a} \tilde{r}^{-1} \tilde{d} \text {. }
$$

We check that, for our base generators, (10) holds with the + sign, from which we conclude, as above, that $(10)$ holds with the + sign throughout. We write this as follows.

$$
\tilde{d} \tilde{c} \tilde{a}=\tilde{r} \tilde{c} \tilde{a} \tilde{r}^{-1} \tilde{d}
$$

The diagonal terms in (11) both yield equation (7). The off diagonal terms both yield the same equation, which we can solve for $\operatorname{coth} \delta$. This yields

$$
\operatorname{coth} \delta=\frac{\cosh \gamma \cosh \mu-\operatorname{coth} \alpha \sinh \gamma \sinh \mu-\sinh \sigma \sinh \rho}{\cosh \sigma \cosh \rho} .
$$

Since $\delta>0$, the left-hand side of the above is necessarily greater than one, while the right-hand side need not be; this yields our last inequality.

$$
\cosh (\rho+\sigma)<\cosh \gamma \cosh \mu-\operatorname{coth} \alpha \sinh \gamma \sinh \mu .
$$

We have shown the following.

Proposition 6.1. Let $R \subset \mathbb{R}^{6}$ be the region defined by the inequalities (2), (3) and (13), where $\mu$ is defined by equation (8) and $\delta$ is defined by equation (12). Then the image of $\Psi$ is contained in $R$.

\section{The NECESSARY CONDITIONS ARE ALSO SUfFICIENT}

In this section, we show the following.

Proposition 7.1. $R$ is equal to the image of $\Psi$.

Proof. We now assume that we have a point $(\alpha, \beta, \gamma, \sigma, \tau, \rho) \in R$, defined by inequalities (2), (3) and (13); we assume that $\mu$ is defined by (8) and that $\delta$ is defined by (12). We write the matrices $\tilde{a}, \ldots, \tilde{d}$ as above, and let $a, \ldots, d$ be the corresponding elements of $\operatorname{PSL}(2, \mathbb{R})$. We need to show that there is a $\phi \in \mathcal{D} \mathcal{F}$, with $(a, \ldots, d)=\left(\phi\left(a_{0}\right), \ldots, \phi\left(d_{0}\right)\right)$.

We have already observed that (3) suffices to ensure that $a$ and $c$ are geometric generators of $K_{1}=\langle a, c\rangle$. We use (7) to define $\nu$, set $r(z)=-\nu \bar{z}$, and we then define $a^{\prime}=\operatorname{rar}^{-1}$. Let $K_{2}=\left\langle a^{\prime}, c\right\rangle$; observe that the left half-plane (right half-plane) is 
precisely invariant under $\langle c\rangle$ in $K_{1}\left(K_{2}\right)$. Hence we can apply the first combination theorem to obtain that $K_{3}=\left\langle K_{1}, K_{2}\right\rangle=\left\langle a, c, a^{\prime}\right\rangle$ is discrete, and represents a sphere with four holes, where $a, a^{\prime}, e$ and $e^{\prime}$ generate the four boundary subgroups (that is; the axis of say $a$ bounds a hyperbolic half-plane that is precisely invariant under the action of $\langle a\rangle$ ). Equations (7) and (8) imply (4), which guarantees that $a^{\prime}=b^{-1} a b$. Since we know the orientation of the axes of $a$ and $a^{\prime}$, and since we know that $b$ is hyperbolic, this can be satisfied only if the axis of $b$ crosses the axes of both $a$ and $a^{\prime}$. It then follows from the second combination theorem that, when we adjoin $b$ to $K_{3}$, we obtain a purely hyperbolic discrete group $K_{4}=\langle a, b, c\rangle$, representing a torus with two equal sized holes. Equations (7) and (12) imply (9), which guarantees that $e^{\prime}=d e d^{-1}$. Inequality (13) guarantees that we can solve (12) for $\delta$ real and positive, and hence that $d$ is hyperbolic. Finally, as above, we can use the second combination theorem to adjoin $d$ to $K_{4}$, to obtain a purely hyperbolic discrete group, $G=\left\langle K_{4}, d\right\rangle=\langle a, b, c, d\rangle$, representing a closed Riemann surface of genus 2 .

Also, since the above construction uses combination theorems in exact analogy with their use in the construction of $G_{0}$, there is a topological deformation of $G_{0}$ onto $G$, where this deformation takes $\left(a_{0}, \ldots, d_{0}\right)$ onto $(a, \ldots, d)$. It follows from our normalization that this deformation is orientation-preserving. It is well known that an orientation-preserving topological deformation can be approximated by a quasiconformal one.

\section{Conclusion}

We gather the above in a single statement.

Theorem 8.1. The mapping $\Psi \circ \Phi: \mathcal{T}_{2} \rightarrow R$ is a real-analytic embedding of the Teichmüller space of surfaces of genus 2 onto the region

$$
R \subset \mathbb{R}^{6}=\{\sinh \alpha, \sinh \beta, \sinh \gamma, \sinh \sigma, \sinh \tau, \sinh \rho\},
$$

defined by inequalities, (2), (3) and (13), where $\mu$ is defined by equation (8) and $\delta$ is defined by equation (12).

8.1. Remark. As remarked above, $\sinh \alpha, \ldots, \sinh \rho$ are algebraic functions of the entries in the matrices and conversely. It follows that these parameters are algebraic if and only if the uniformizing group $G$, which is a discrete faithful representation of $G_{0}$, is a subgroup of some $\operatorname{PSL}(2, \mathbf{k})$, where $k$ is a (real) number field.

\section{REFERENCES}

[1] J. Gilman and B. Maskit. An algorithm for 2-generator fuchsian groups. Mich. Math. J., 38:13-32, 1991. MR 92f:30062

[2] A. Haas and P. Susskind. The geometry of the hyperelliptic involution in genus two. Proc. Amer. Math. Soc., 105:159-165, 1989. MR 89e:30078

[3] B. Maskit. Explicit matrices for fuchsian groups. Cont. Math., 169:451-466, 1994 . MR 96f: 30045

[4] B. Maskit. A picture of moduli space. Invent. math., 126:341-390, 1996. MR 97m:32034

Department of Mathematics, The University at Stony Brook, Stony Brook, New YORK 11794-3651

E-mail address: bernie@math.sunysb.edu 